\title{
Relative figure of merit of optical interferometry and spectroscopy.
}

\section{Example of parameter estimates of P Cygni circumstellar envelope}

\section{M.S. Burgin ${ }^{1,2}$ and A. Chalabaev ${ }^{2}$}

${ }^{1}$ Astro-Space Center FIAN, Profsoyuznaya 84/32, 117810 Moscow, Russia

e-mail: mburgin@dpc.asc.rssi.ru

${ }^{2}$ CNRS, Laboratoire d'Astrophysique, Observatoire de Grenoble, UMR 5571, BP. 53X, 38041 Grenoble Cedex, France e-mail: Almas.Chalabaev@obs.ujf-grenoble.fr

Astron. Astrophys. Suppl. Ser. 142 (2000) 325-338

The on-plot labels indicating the sets of adjustible, nuisance, and target parameters are missing from Figs. 2-6. In what follows, the missing text is specified for each figure. The numbers in parentheses identify the relevant individual plot, the order being from topmost plot to bottom.

Fig. 2. (1) $\alpha$; (2) $\dot{M} ;$ (3) $v_{\mathrm{c}}$; (4) $T_{1}$.

Fig. 3. (1) $\alpha, T_{1}$; (2) $\alpha, v_{\mathrm{c}}, T_{1}$; (3) $\alpha, v_{\mathrm{c}}, T_{1}, \Delta_{2} T$; (4) $\alpha, v_{\mathrm{c}}, T_{1}, \Delta_{2} T, \Delta_{3} T$.

Fig. 4. (1) $\alpha$; (2) $v_{\mathrm{c}}$; (3) $T_{1}$; (4) $\Delta_{2} T$; (5) $\Delta_{3} T$.

Fig. 5. (1) $\alpha, \dot{M}, v_{\mathrm{c}}$; (2) $T_{1}, \Delta_{2} T, \Delta_{3} T$; (3) $\alpha, T_{1}, \Delta_{2} T$; (4) $\alpha, \dot{M}, T_{1}$; (5) $\alpha, \dot{M}, v_{\mathrm{c}}, T_{1}, \Delta_{2} T, \Delta_{3} T$.

Fig. 6. (1) $\alpha$; (2) $v_{\mathrm{c}}$; (3) $T_{1}$; (4) $\Delta_{2} T$; (5) $\Delta_{3} T$; (6) $\Delta_{4} T$; (7) $\Delta_{5} T$.

\section{Destriping of polarized data in a CMB mission with a circular scanning strategy}

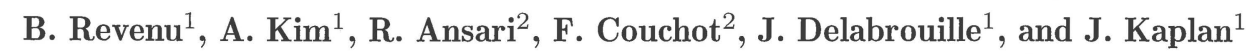

${ }^{1}$ Physique Corpusculaire et Cosmologie, Collège de France, 11 place Marcelin Berthelot, 75231 Paris Cedex 05, France

${ }^{2}$ Laboratoire de l'Accélérateur Linéaire, IN2P3 CNRS, Université Paris Sud, 91405 Orsay, France

Astron. Astrophys. Suppl. Ser. 142 (2000) 499-509

New caption of Fig. 8: The Mollweide projection of the residuals of the $I$-Stokes parameter after destriping of $1 / f$ noise plus white noise with $\eta=1$

New caption of Fig. 9: The Mollweide projection of the residuals of the $I$-Stokes parameter after zeroing the average of the circles, for $1 / f$ noise plus white noise with $\eta=1$

New caption of Fig. 10: The Mollweide projection of the residuals of the $Q$-Stokes parameter for a white noise mission 